\title{
HD 50975: a yellow supergiant in a spectroscopic binary system ${ }^{\star}$
}

\author{
J. Sperauskas ${ }^{1}$, L. Začs ${ }^{1,2}$, S. Raudeliūnas ${ }^{1}$, F. Musaev ${ }^{3,4,5}$, and V. Puzin ${ }^{3}$ \\ 1 Vilnius University Observatory, Ciurlionio 29, Vilnius 03100, Lithuania \\ e-mail: zacs@latnet.lv \\ 2 Laser Center, University of Latvia, Raiņa bulvāris 19, 1586 Rīga, Latvia \\ 3 Institute of Astronomy of the Russian AS, 48 Pyatnitskaya st., 119017 Moscow, Russia \\ ${ }^{4}$ Terskol Branch of Institute of Astronomy of the Russian AS, 361605 Peak Terskol, Kabardino-Balkaria, Russia \\ 5 Special Astrophysical Observatory of the Russian AS, 369167 Nizhnij Arkhyz, Russia
}

Received 21 October 2013 / Accepted 4 August 2014

\begin{abstract}
Context. Recent detection of a yellow supergiant star as a possible progenitor of a supernova has posed serious questions about our understanding of the evolution of massive stars.

Aims. The spectroscopic binary star HD 50975 with an unseen hot secondary was studied in detail with the main goal of estimating fundamental parameters of both components and the binary system.

Methods. A comprehensive analysis and modeling of collected long-term radial velocity measurements, photometric data, and spectra was performed to calculate orbital elements, atmospheric parameters, abundances, and luminosities. The spectrum in an ultraviolet region was studied to clarify the nature of an unseen companion star.

Results. The orbital period was found to be $190.22 \pm 0.01$ days. The primary star (hereafter HD 50975A) is a yellow supergiant with an effective temperature $T_{\text {eff }}=5900 \pm 150 \mathrm{~K}$ and a surface gravity of $\log (g)=1.4 \pm 0.3(\mathrm{cgs})$. The atmosphere of HD 50975 A is slightly metal deficient relative to solar, $[\mathrm{Fe} / \mathrm{H}]=-0.26 \pm 0.06$ dex. Abundances of $\mathrm{Si}$ and $\mathrm{Ca}$ are close to the scaled solar composition. The r-process element europium is enhanced, $[\mathrm{Eu} / \mathrm{H}]=+0.61 \pm 0.07$. The bolometric magnitude of the primary was estimated to be $M_{\text {bol }}=-5.5 \pm 0.3 \mathrm{mag}$ and its mass to be $10.7 \pm 2.0 M_{\odot}$. The secondary (hereafter HD 50975B) is a hot star of spectral type $\sim \mathrm{B} 2 \mathrm{near}$ ZAMS with an effective temperature of $T_{\text {eff }} \simeq 21000 \mathrm{~K}$ and a mass $M \simeq 8.6 M_{\odot}$. The distance between HD 50975A and B is about $370 R_{\odot}$. The binary star is near a semi-detached configuration with a radius, $R_{\mathrm{A}} \simeq 107 R_{\odot}$, and a radius of Roche lobe of about $120 R_{\odot}$ for the primary star.
\end{abstract}

Key words. binaries: spectroscopic - stars: evolution - stars: fundamental parameters - stars: individual: HD 50975 stars: abundances

\section{Introduction}

Recent detection of yellow supergiant star as the possible progenitor of supernovae have raised serious questions about our understanding of the evolution of massive stars. Bersten et al. (2012) and Benvenuto et al. (2013) present recent evidence that the yellow supergiant star found at the location of supernova SN 2011dh in the famous nearby galaxy M51 was the SN progenitor. The authors of these papers have produced a self-consistent model to explain how the star exploded. In their model, the exploding massive star was a member of a close binary system. A comprehensive analysis of similar binary systems will advance our knowledge of binary systems as possible progenitors of supernova explosions.

The spectrum of HD 50975 was classified as a composite spectrum of two stars, F8 Ib supergiant and an early type star, using the objective prism with dispersion of $108 \AA \mathrm{Am}^{-1}$ at $\mathrm{H}_{\gamma}$ by MacConnell \& Bidelman (1976). The same spectral type was found for a primary star by Barbier et al. (1978) on the basis of spectra with dispersion of $200 \AA \mathrm{mm}^{-1}$. Barbier et al. (1978) analyzed photometric data gathered using the ultraviolet

\footnotetext{
* The reduced spectra are only available at the CDS via anonymous ftp to cdsarc.u-strasbg. fr (130.79.128.5) or via http://cdsarc.u-strasbg.fr/viz-bin/qcat?]/A+A/570/A3
}

satellite TD1 and noted that HD 50975 is an "unexplained case". HD 50975 is located in the region of G4 subdwarfs according to uvby photometry (Olsen 1979), and low-amplitude light variations are suspected by Olsen (1983). The period $(P)$ and amplitude $(A)$ of light variations were determined by Koen \& Eyer (2002), $P=95.1$ days and $A=0.013 \mathrm{mag}$. Radial velocity variations were detected for HD 50975 by Bartkevicius \& Sperauskas (1994).

In the present paper a comprehensive analysis and modeling of the collected long-term radial velocity measurements, photometric data, spectral energy distribution (SED), cross-correlation function (CCF), and high-resolution spectra was performed for HD 50975 to calculate orbital elements, atmospheric parameters, abundances, luminosities, and masses. A spectrum in the ultraviolet and blue wavelength region was analyzed in detail to clarify the nature of unseen companion.

\section{Observations}

Radial-velocity monitoring of HD 50975 was started in 1989 using the CORAVEL spectrometer of the Vilnius University installed on the $1.65 \mathrm{~m}$ and $0.63 \mathrm{~m}$ telescopes at the Moletai Observatory (Lithuania). The CORAVEL spectrometer (Upgren et al. 2002) is based on the principles of the photoelectric radialvelocity scanner developed by Griffin (1967) and operates by 
scanning a spectrum of star across the mask and obtaining an online cross-correlation velocity. The standard deviation of a single observation for late-type stars brighter than about the 11th magnitude is usually better than $0.8 \mathrm{~km} \mathrm{~s}^{-1}$. The velocities have been standardized using the observations of IAU radial velocity standards (Udry et al. 1999) and are close to the system of velocities published by Nidever et al. (2002). The difference in zero point was found to be $0.14 \mathrm{~km} \mathrm{~s}^{-1}$ for F-G-K type stars and exhibit an rms scatter of $0.5 \mathrm{~km} \mathrm{~s}^{-1}$.

High-resolution spectra for HD 50975 were obtained with the coudé échelle spectrometer MAESTRO on the $2 \mathrm{~m}$ telescope at the Observatory on the Terskol Peak in Northern Caucasus (altitude of $3100 \mathrm{~m}$ ) equipped with a Wright Instruments CCD detector. Three spectra were obtained between November 2012 and January 2013. In addition, spectra of several hot, rapidly rotating stars were observed to identify the telluric absorption lines. All the spectra covered region from about 3600 to $9600 \AA$ and have a resolving power $R \sim 45000$. Unfortunately, our efforts to observe a high-resolution spectrum shortward of $3600 \AA$ was unsuccessful. All spectra were bias-subtracted, flat-field-corrected, and converted to one-dimensional spectra using the standard DECH20T package ${ }^{1}$. The equivalent widths of absorption lines were measured by Gaussian fitting to the observed profiles.

\section{Analysis and results}

\subsection{Orbital solution and light curve}

Orbital parameters for HD 50975 were calculated using 53 radial velocities observed in the time span from February 1989 to April 2008. The measured heliocentric radial velocities and their errors are presented in Table 1, together with the time of observations, phases, and residuals $(\mathrm{O}-\mathrm{C})$. Orbital elements are listed in Table 2. All of the radial velocities upon which the orbit is based have been attributed the same (unit) weight. Radial velocity curve for HD 50975A is shown in Fig. 1 (upper panel). In addition, two light curves are plotted in the middle and bottom panels. Published photometric data are phased with the orbital period of $P=190.22 \mathrm{~d}$ and $T_{\max }$ of radial velocity as an epoch for phase $=0$. The photometric data were retrieved from the HIPPARCOS and ASAS databases: 74 measurements taken from the HIPPARCOS epoch photometry (Perryman $\&$ ESA 1997) and 410 measurements from the ASAS-3 $V$ band photometry (Pojmanski 2002). We used ASAS photometry observed by a two-pixel aperture because of the lowest reduction errors and scatter of measurements. In addition, the measurements of $D$ (uncertain) quality and crude errors ( $3 \sigma$ threshold) were rejected. The remaining 389 measurements were phased with the orbital period and are plotted in Fig. 1 (middle panel). Double-peaked light curve synchronized with the maximum and minimum of radial velocity variations gives evidence about the ellipsoidal shape of the primary star.

\subsection{IUE spectra}

The spectrum of the hot secondary star dominates the composite spectrum of HD 50975 in the far ultraviolet spectral region. Low-dispersion spectra observed by SWP and LWR cameras (SWP 04082 and LWR 03613) are available for HD 50975 in the IUE archive. We used IUE NEWSIPS data recalibrated by Massa \& Fitzpatrick (2000) in order to minimize systematic errors. To avoid the $2200 \AA$ interstellar extinction bump and

\footnotetext{
1 http://www . gazinur . com/Spectra-Processing.html
}

Table 1. Radial velocity measurements for HD 50975A, along with the uncertainties, phases, and residuals for the orbital curve.

\begin{tabular}{|c|c|c|c|c|c|}
\hline $\begin{array}{l}\text { HJD-2 } 400000 \\
\text { days }\end{array}$ & Epoch & Phase & $\begin{array}{r}V r \\
\mathrm{~km} \mathrm{~s}^{-1}\end{array}$ & $\begin{array}{l}(\mathrm{O}-\mathrm{C}) \\
\mathrm{km} \mathrm{s}^{-1}\end{array}$ & $\begin{array}{c}\sigma \\
\mathrm{km} \mathrm{s}^{-1}\end{array}$ \\
\hline 47572.171 & 37 & .598260 & 0.30 & -1.161 & 0.6 \\
\hline 47575.173 & 37 & .614042 & 3.40 & -0.602 & 0.5 \\
\hline 47924.292 & 35 & .449374 & -3.10 & 1.008 & 0.6 \\
\hline 47935.288 & 35 & .507181 & -5.10 & 1.044 & 0.6 \\
\hline 47942.190 & 35 & .543465 & -5.60 & -0.949 & 0.6 \\
\hline 47950.213 & 35 & .585642 & 0.50 & 0.832 & 0.5 \\
\hline 48302.251 & 33 & .436320 & -3.60 & -0.686 & 0.5 \\
\hline 48305.242 & 33 & .452044 & -3.40 & 0.920 & 0.7 \\
\hline 48315.201 & 33 & .504399 & -6.70 & -0.530 & 0.6 \\
\hline 48319.283 & 33 & .525858 & -4.00 & 1.641 & 1.2 \\
\hline 48321.260 & 33 & .536251 & -5.60 & -0.484 & 0.8 \\
\hline 48334.238 & 33 & .604477 & 2.80 & 0.376 & 0.5 \\
\hline 48347.220 & 33 & .672724 & 16.80 & 0.893 & 0.5 \\
\hline 48349.228 & 33 & .683280 & 18.20 & -0.178 & 0.6 \\
\hline 48351.208 & 33 & 693689 & 20.80 & -0.087 & 0.6 \\
\hline 48656.284 & 31 & .297486 & 23.30 & 0.237 & 0.6 \\
\hline 48658.228 & 31 & .307706 & 20.90 & 0.353 & 0.9 \\
\hline 48660.281 & 31 & .318498 & 17.90 & -0.057 & 0.8 \\
\hline 48663.278 & 31 & .334254 & 14.70 & 0.380 & 0.8 \\
\hline 48665.213 & 31 & .344426 & 12.30 & 0.221 & 1.0 \\
\hline 48666.198 & 31 & .349604 & 12.10 & 1.126 & 0.6 \\
\hline 48670.200 & 31 & .370643 & 6.60 & -0.160 & 0.6 \\
\hline 48672.196 & 31 & .381136 & 4.10 & -0.740 & 0.6 \\
\hline 48676.272 & 31 & .402564 & -0.20 & -1.538 & 0.8 \\
\hline 49038.222 & 29 & .305349 & 19.60 & -1.522 & 0.7 \\
\hline 49042.276 & 29 & .326661 & 16.40 & 0.351 & 0.7 \\
\hline 49046.189 & 29 & .347232 & 11.90 & 0.423 & 0.6 \\
\hline 49050.206 & 29 & .368350 & 5.20 & -1.997 & 0.7 \\
\hline 49058.201 & 29 & .410380 & 0.00 & -0.210 & 0.7 \\
\hline 49061.262 & 29 & .426472 & -1.90 & -0.057 & 0.7 \\
\hline 49065.239 & 29 & .447379 & -3.70 & 0.242 & 0.5 \\
\hline 51268.292 & 17 & .028917 & 76.40 & 0.414 & 0.7 \\
\hline 51269.271 & 17 & .034064 & 75.90 & 0.177 & 0.9 \\
\hline 51271.289 & 17 & .044673 & 75.20 & 0.153 & 0.7 \\
\hline 51892.441 & 14 & .310094 & 19.70 & -0.268 & 0.7 \\
\hline 51998.252 & 14 & .866347 & 62.00 & -0.899 & 1.1 \\
\hline 51999.289 & 14 & .871798 & 63.10 & -0.839 & 0.7 \\
\hline 52008.281 & 14 & .919069 & 70.10 & -1.326 & 0.8 \\
\hline 53794.357 & 4 & .308543 & 20.50 & 0.156 & 0.7 \\
\hline 53816.280 & 4 & .423793 & -1.60 & -0.073 & 0.6 \\
\hline 54056.032 & 3 & .684179 & 18.50 & -0.092 & 0.4 \\
\hline 54076.853 & 3 & .793636 & 46.40 & -0.057 & 0.4 \\
\hline 54083.875 & 3 & .830551 & 55.10 & -0.224 & 0.5 \\
\hline 54096.843 & 3 & .898724 & 68.90 & 0.340 & 0.5 \\
\hline 54447.627 & 1 & .742810 & 33.70 & 0.330 & 0.5 \\
\hline 54469.483 & 1 & .857708 & 61.60 & 0.415 & 0.6 \\
\hline 54470.521 & 1 & .863164 & 62.10 & -0.177 & 0.4 \\
\hline 54471.444 & 1 & .868017 & 64.10 & 0.879 & 0.6 \\
\hline 54512.423 & 0 & .083445 & 71.40 & 0.297 & 0.5 \\
\hline 54513.327 & 0 & .088197 & 70.70 & 0.232 & 0.4 \\
\hline 54530.362 & 0 & .177751 & 53.80 & 0.393 & 0.4 \\
\hline 54531.315 & 0 & .182761 & 51.70 & -0.527 & 0.6 \\
\hline 54580.289 & 0 & .440219 & -3.30 & -0.003 & 0.4 \\
\hline
\end{tabular}

the possible influence of primary star HD 50975A we employed only spectra observed by SWP camera. In addition, spectra of four comparison stars of spectral types between B1 and B4 were extracted from the database. Normalized spectra are plotted in Fig. 2. Criteria of spectral classification developed by Rountree \& Sonneborn (1991) in the far ultraviolet region are used for spectral classification of the secondary star HD 50975B. On the basis of temperature sensitive features of Si II at $1260 \AA$, Si III 


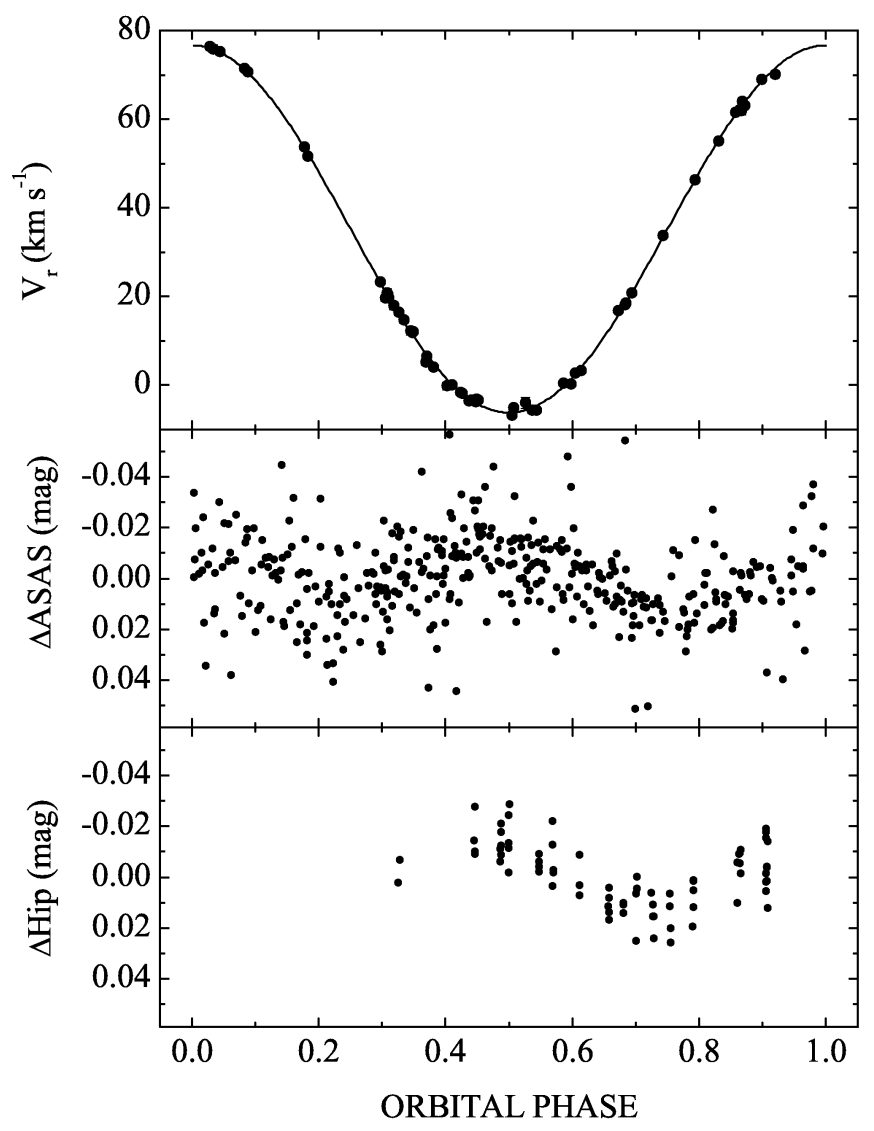

Fig. 1. Observed heliocentric radial velocities for HD 50975A (filled circles) as a function of phase, with the velocity curve corresponding to the calculated orbital elements (upper panel). ASAS (middle panel) and HIPPARCos (lower panel) photometry phased using the same orbital period gives evidence of the ellipsoidal shape of the primary star.

Table 2. The orbital elements along with standard deviations for HD 50975A.

\begin{tabular}{lc}
\hline \hline Orbital period & $P_{\mathrm{A}}=190.22 \pm 0.01$ days \\
Center-of-mass velocity & $V_{0}=35.24 \pm 0.08 \mathrm{~km} \mathrm{~s}^{-1}$ \\
Half-amplitude & $K_{\mathrm{A}}=41.4 \pm 0.1 \mathrm{~km} \mathrm{~s}^{-1}$ \\
Eccentricity & $e=0$ (adopted) \\
Projected semimajor axis & $a_{\mathrm{A}} \sin (i)=(1.084 \pm 0.003) 10^{8} \mathrm{~km}$ \\
Function of the mass & $f(m)=1.402 \pm 0.005 M_{\odot}$ \\
Time of maximum velocity (HJD) & $T_{\max \mathrm{A}}=2454496.55 \pm 0.08$ \\
Standard deviation of residuals & $\sigma(\mathrm{O}-\mathrm{C})_{\mathrm{A}}=0.7 \mathrm{~km} \mathrm{~s}^{-1}$ \\
\hline
\end{tabular}

at $1300 \AA, \mathrm{C}$ II at $1340 \AA$, and Si IV at $1400 \AA$, the temperature subtype was found to be $\mathrm{B} 2 \pm 0.5$ for HD $50975 \mathrm{~B}$.

The profile of hydrogen Lyman $\alpha$ line was synthesized using code STARSP and Kurucz (1993) models (see Sect. 3.6.3) for the set of effective temperatures between 15000 and $25000 \mathrm{~K}$. The best fit between the observed and calculated profiles (see Fig. 2) was found for the atmospheric model with an effective temperature, $T_{\text {eff }}=17000 \mathrm{~K}$, and gravity, $\log (g)=4.5(\mathrm{cgs})$, in support of spectral type $\sim$ B3 for HD 50975B.

\subsection{The uvby photometry}

A set of $u v b y$ colors for the primary component HD 50975A was calculated using unreddened colors of HD 50975 (Hauck $\&$ Mermilliod 1998) for a range of color excesses $E(B-V)$

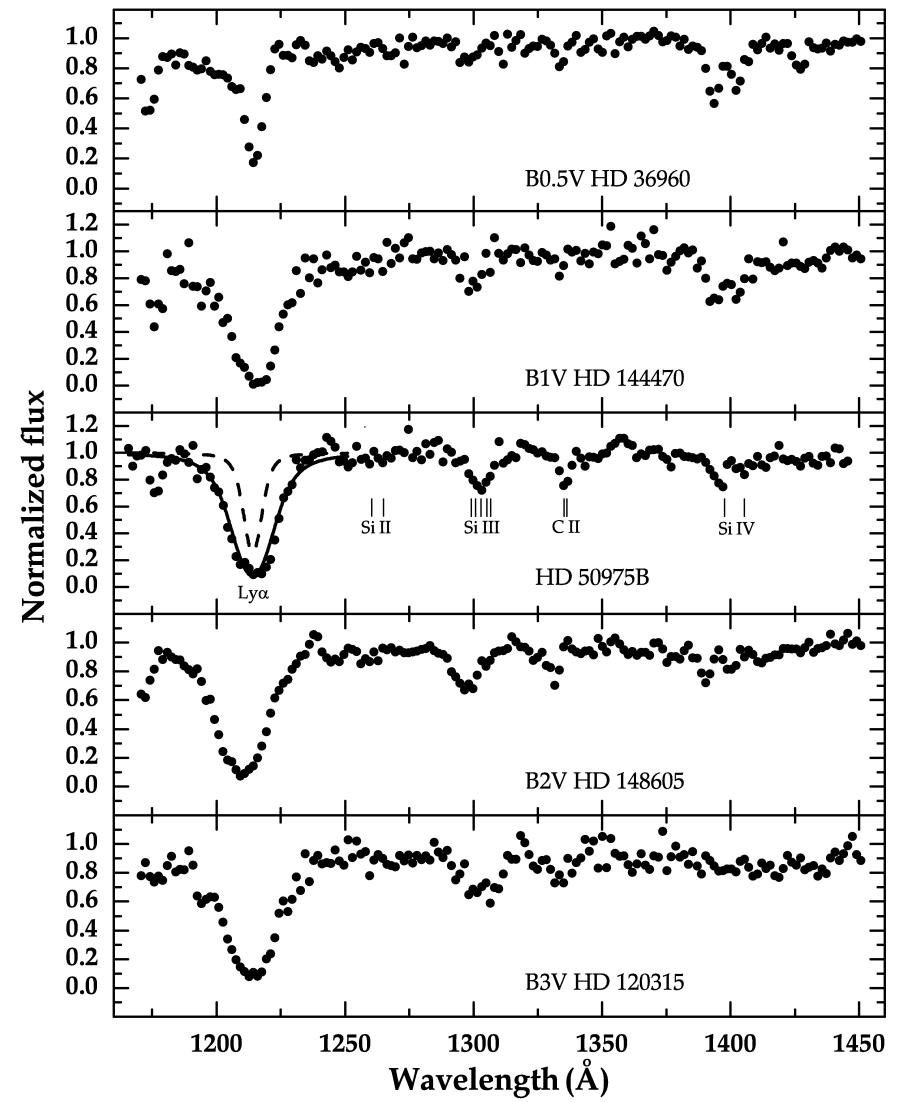

Fig. 2. Spectrum of HD 50975 retrieved from IUE archive in the far ultraviolet region where radiation of the hot secondary star dominates. Four standard stars of spectral types between B 0.5 and B 3 are presented for comparison purposes. The profiles of Lyman $\alpha$ line at $1215 \AA$ calculated using atmospheric models with $T_{\text {eff }}=17000$ (solid line) and $25000 \mathrm{~K}$ (dashed line) are shown.

from 0.05 to $0.25 \mathrm{mag}$. For $(b-y), m_{1}$, and $c_{1}$ of HD 50975A, the folowing limits are accepted:

1) The range of $(b-y)$ values from $0.34-0.44$ mag according mean colors for F7-F9 supergiants (Gray et al. 2001), which correspond the uncertainty in spectral subtype \pm 1 . This range was increased by 0.02 mag because of possible errors in observations and reduction.

2) The values of $c_{1}$ and $m_{1}$ are within \pm 0.1 mag for the standard line of supergiants (Gray 1991). The appropriate contribution of the secondary star was subtracted, and the difference in absolute magnitudes between primary and secondary components was varied in steps of 0.01 mag. Normal ubvy colors for the secondary star were adopted from Crawford (1978). In addition, the relation $(b-y)_{0}=0.0867\left(c_{1}+\right.$ $\left.m_{1}\right)+0.125$ given by Shulov (1986) was used to calculate the normal $(b-y)$ index for B star because Crawford (1978) provided only $m_{1}$ and $c_{1}$ indices. The results of calculations are plotted in Fig. 3 with the sequence of yellow supergiants given by Gray (1991) and the main sequence F-type stars given by Crawford (1975). In addition, a sample of supergiants of spectral types from F6 to G0 classified by Gray et al. (2001) are plotted for comparison purposes. Their colors are adopted from the photometric catalog compiled by Hauck \& Mermilliod (1998) and are unreddened using $E(b-y)$ values given by Gray et al. (2001) and reddening ratios given by Crawford \& Mandwewala (1976). The range 


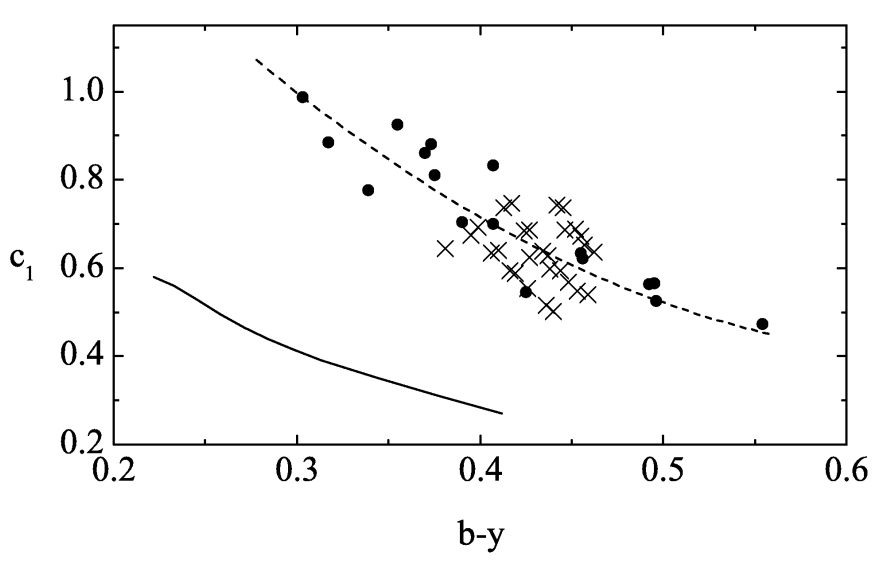

Fig. 3. Modeled position of HD 50975A (crosses) in the $c_{1}-(b-y)$ diagram calculated for the set of parameters (spectral type, magnitude, extinction) of the secondary star. The solid line represents main sequence F stars; dashed line - sequence of yellow supergiants; filled circles - a sample of single supergiants of spectral types from F6 to G0.

of parameters for the secondary star was found to be as follows: spectral type B0.5 V-B1.5 V, difference in magnitudes between primary and secondary star, $\Delta V=1.8-2.4 \mathrm{mag}$, and extinction, $E(B-V)=0.1-0.18 \mathrm{mag}$.

\subsection{Spectral energy distribution}

A set of the composite SEDs of two stars was calculated in the spectral region from 1250 to $5450 \AA$ using the SEDs of MK spectral types given in Sviderskiene (1988) and was compared with the observed SED of HD 50975, which is based on the IUE spectrum and uvby photometry. The IUE spectrum was averaged over $50 \AA$ intervals centered on the same wavelength as for MK-type SED. The uvby magnitudes were converted into absolute fluxes using the Gray (1998) calibration. The SED of MK types expressed in terms of intensities per unit wavelength interval were transformed into absolute fluxes by equating intensities to fluxes in the y passband $\left(\lambda_{0}=5456 \AA\right)$ of the uvby system. Assuming a spectral type of F8 I for the primary star HD 50975A, the best fit between observed and calculated SED was achieved for the secondary of spectral type B1 V, a ratio of fluxes of 0.12 at $5500 \AA$ for the companion stars, and $E(B-V)=0.12$ mag (see Fig. 4). We also estimated extinction using IUE data and the relationship of Vieira \& Ponz (1995) applicable for O3-G5 single stars:

$E(B-V)=1.368 \log \left[\left(225 F_{1}-575 F_{2}\right) / 800 F_{\mathrm{c}}\right]$,

where $F_{1}, F_{2}, F_{\text {c }}$ are fluxes estimated at $\lambda_{1}=1600 \AA, \lambda_{2}=$ $2400 \AA, \lambda_{\mathrm{c}}=2175 \AA$, respectively. The contribution of F8 Ib star to the flux in the ultraviolet (up to $1 \%$ at $1600 \AA, 3-5 \%$ at $2175 \AA$, and $5-8 \%$ at $2400 \AA$ ) was extracted. The extinction was found to be $E(B-V)=0.14 \pm 0.04$ using this method for both small and large apertures and for the averaging windows of $50 \AA$.

\subsection{Coravel cross-correlation function}

The shape of CCF measured with the CORAVEL spectrometer is a convolution of averaged profiles of absorption lines and slots of mask on which the spectrum of star was focused. Therefore, the area defined by CCF is an analogue for an equivalent width

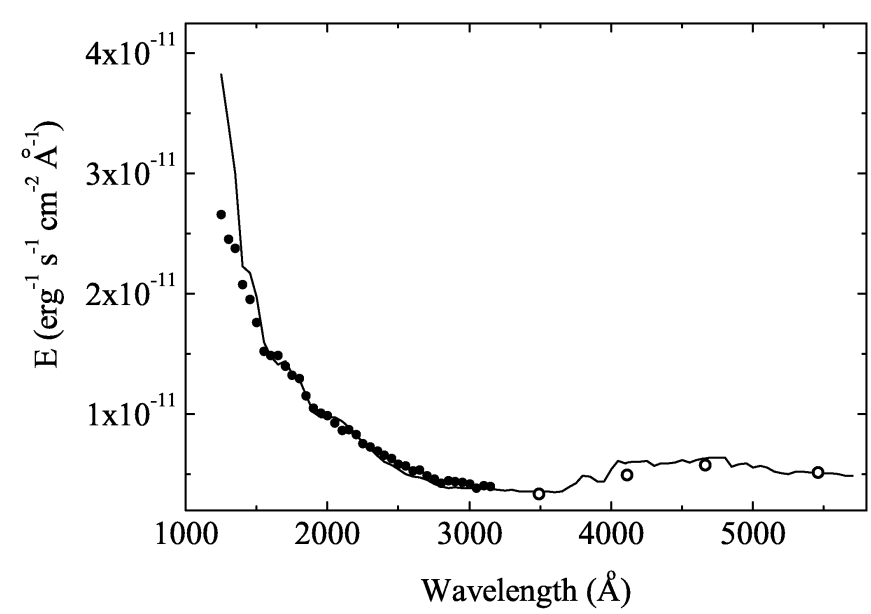

Fig. 4. Comparison of the observed (IUE observations: filled circles, $u v b y$ photometry unreddened by $E(b-v)=0.12$ : open circles), and modeled SED for HD 50975. The solid line represents a composite SED of two stars of spectral type F8 Iab and B1 V with a ratio of fluxes of 0.12 at $5500 \AA$.

(EW) of spectral line. The continuum contribution from the hot secondary partially fill the photospheric lines of the primary, making them less deep than they should be. This extra contribution (so-called veiling) can be measured by adding an extra continuum to a template star in order to fit the observed lines. Veiling of the spectrum of primary star reduces the equivalent widths. The decrease in EW can be used to estimate a difference in magnitudes between primary and unseen secondary components. Because of the different SEDs of the companion stars, the amount of veiling of the primary star increases toward short wavelengths.

The CCF of HD 50975 are plotted in Fig. 5, along with those for one main sequence star of spectral type G0V and single supergiant of spectral type F8 Ib (HD 194093), i.e. the same spectral type as for HD 50975. The spectral type of a supergiant was estimated by Gray et al. (2001). The EW of CCF for HD 50975 is about $15 \%$ lower than measured for the F8 Ib comparison star. Numerical simulations of the cross-corelation between the spectrum of F8 Ib, the B2 star, and the spectral transmission function of the mask was carried out. The decrease in EW by $15 \%$ corresponds to the difference of about 2.5 mag in magnitude between the primary and secondary stars in the case of similar metallicity of HD 50975 and the comparison star.

\subsection{High-resolution spectroscopy}

Absorption lines of iron are selected and measured in the observed high-resolution spectrum of HD 50975 with the goal of estimating the atmospheric parameters and metallicity of the primary star HD 50975A. The red and near infrared spectral region from about 6100 to $9600 \AA$ was exploited to avoid a significant contribution of the secondary star. The modeled and scaled flux, $F_{v}(B 2) / F_{v}(F 8)=0.1$ at $5500 \AA$, of the companion stars limits the contribution of the flux of B2 star below $8 \%$ in the red and near-infrared wavelength region $(6.5 \pm 1.5 \%$ from 6000 to $8000 \AA$ ). Such veiling should cause a minor decrease in EWs and a calculated metallicity $(<0.1 \mathrm{dex})$. In addition, unblended iron lines are measured in the visual and blue spectral regions with the goal of quantiting the amount of veiling in different spectral regions. 


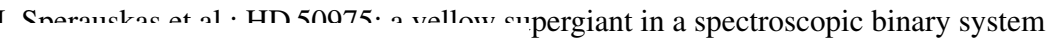

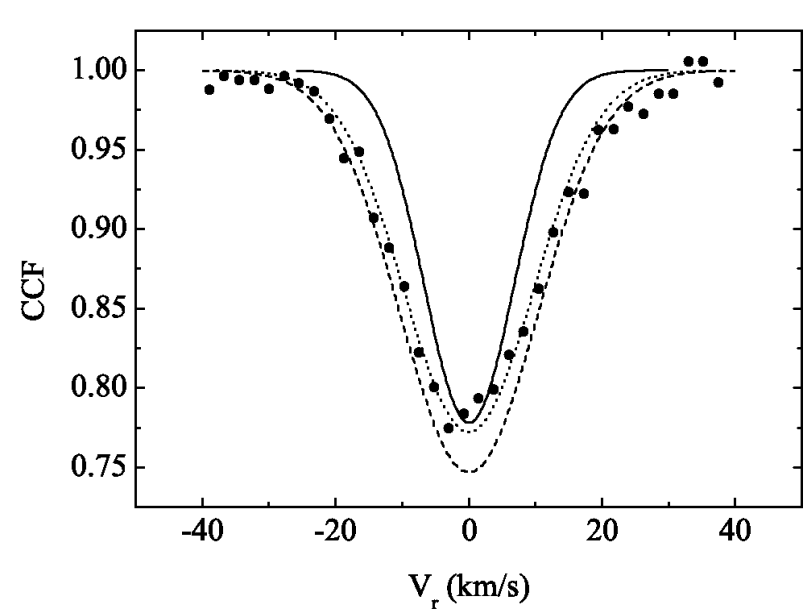

Fig. 5. Cross-corelation function measured using CORAVEL spectrometer for HD 50975 (filled circles), along with those for two comparison stars: G0 V main sequence star (solid line) and single F8 I supergiant (dashed line). The dotted line represents CCF of the single F8 I star decreased in the equivalent width by $15 \%$.

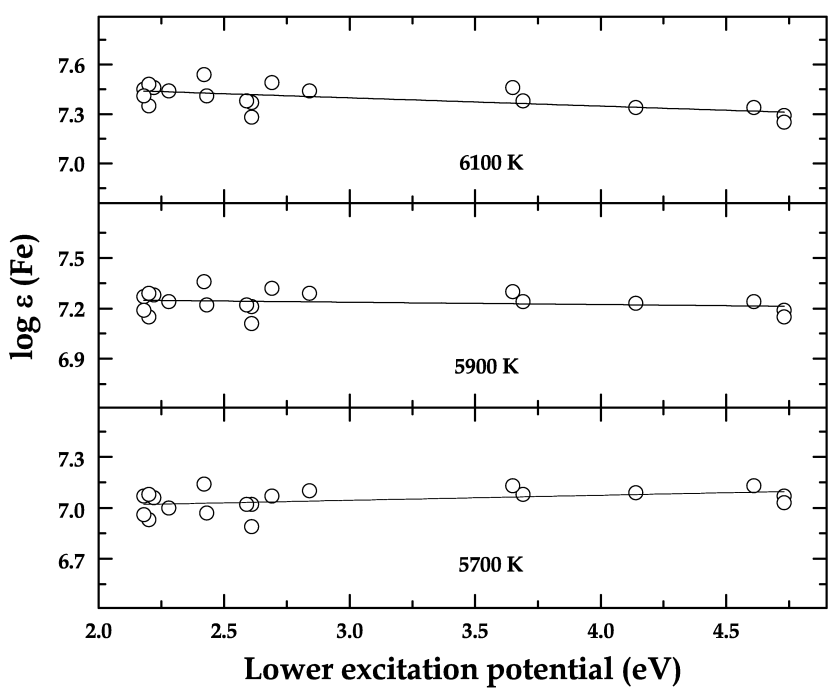

Fig. 6. Abundance of iron derived from the Fe I lines in red wavelength region vs. the excitation potential of the lower energy level of the line, illustrating the estimation of the excitation temperature for HD 50975A.

A search for absorption lines for the secondary star was performed in the blue wavelength region shortward of $4500 \AA$. Unfortunately, in the blue region the spectrum of primary star is very crowded by absorption lines and detection of weak lines of the secondary star was plagued. A high-resolution spectrum shortward of $3600 \AA$ is needed to detect photospheric lines of B2 star and to calculate the mass ratio of components.

\subsubsection{Effective temperature of primary star}

The excitation balance of iron was analyzed to obtain a colorindependent estimate of the temperature, and $T_{\text {eff }}$ was chosen so that the derived iron abundance vs. excitation potential has a zero slope (Fig. 6). This excitation analysis confirms a temperature $T_{\text {eff }}=5900 \pm 150 \mathrm{~K}$ for HD 50975A, which is close to the mean effective temperature of F8 I supergiants, $T_{\text {eff }}=5800 \mathrm{~K}$ (see Lyubimkov et al. 2010).

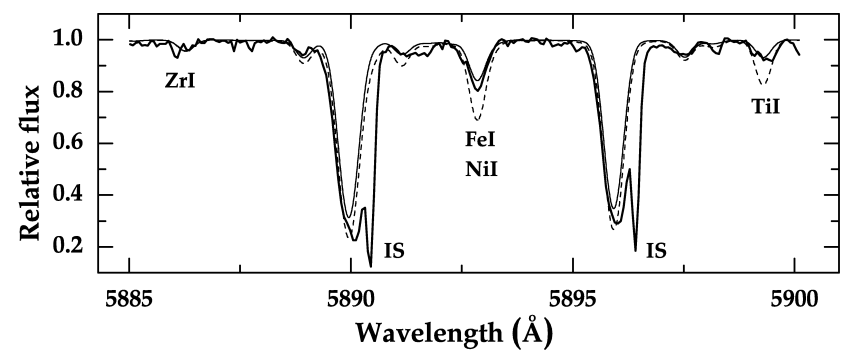

Fig. 7. Observed spectrum of HD50975 (thick line) around the $\mathrm{Na}$ I D12 doublet, along with the calculated photospheric spectra for two different effective temperatures, $T_{\text {eff }}=6000 \mathrm{~K}$ (thin line) and $5500 \mathrm{~K}$ (dashed line). The wavelengths are corrected for stellar velocity. Sharp interstellar components are visible on the red wings of the photospheric lines.

The intensity of the sodium D12 doublet is a good indicator of stellar effective temperature; however, the sodium photospheric lines are asymmetric probably because of unresolved circumstellar absorption, hence plaguing examination of temperature using this criterion. In addition, the interstellar component is visible on the red wings of D1 and D2. The results of spectral synthesis for two effective temperatures and for the final calculated gravity, microturbulence, and abundances are presented in Fig. 7. As can be seen, the synthesized profiles of photospheric lines confirm an effective temperature of HD 50975A higher than $5500 \mathrm{~K}$. The EW was measured to be $250 \mathrm{~m} \AA$ for interstellar D1 component, and an independent estimate of reddening was made for HD 50975 using relation between equivalent width and reddening (see Munari \& Zwitter 1997), $E(B-V) \simeq 0.09 \pm 0.01 \mathrm{mag}$.

\subsubsection{Gravity and microturbulence}

The available spectroscopic methods for estimating the surface gravity are based on the study of the ionization equilibrium and damping wings of strong lines. Gravity for the primary star HD 50975A was determined by requiring that Fe I and Fe II lines provide the same iron abundance (see Fig. 8). From the ionization balance, $\log g=1.4 \pm 0.3$ (cgs) was found. The wings of the $\mathrm{H}_{\alpha}$ line synthesized using Kurucz (1993) model in general confirm the gravity calculated using ionization balance (see Fig. 9).

The procedure for estimating the microturbulent velocity is illustrated in Fig. 8 for the primary star of HD 50975. As seen, a good agreement between the iron abundances derived from individual lines was found using $\xi_{\mathrm{t}}=4.1 \pm 0.3 \mathrm{~km} \mathrm{~s}^{-1}$. The final atmospheric parameters for HD 50975A are given in Table 3.

\subsubsection{Abundances}

The abundances were computed using the standard LTE line analysis program WIDTH6, written by Kurucz. This code employs an input model atmosphere to compute the strength of a given atomic line formed in such an atmosphere. The model atmospheres were taken from the grid of Kurucz (1993). The main goal was to estimate the iron abundance in the atmosphere of HD 50975. In addition, we calculated abundances for chemical elements that are represented by a set of unblended lines redward of $6100 \AA$. Spectral lines stronger than $300 \mathrm{~m} \AA$ were not used for abundance calculations. Oscillator strengths for the lines are compiled from a variety of sources by Luck (priv. comm.). A majority of the values are from high-precision 
Table 3. Final atmospheric parameters and relative (to the Sun) abundances for primary star HD 50975A calculated using lines in the red and near-infrared wavelength region.

\begin{tabular}{lcccccccc}
\hline \hline $\begin{array}{l}T_{\text {eff }} \\
(\mathrm{K})\end{array}$ & $\begin{array}{c}\log g \\
(\mathrm{cgs})\end{array}$ & $\begin{array}{c}\xi_{\mathrm{t}} \\
\left(\mathrm{km} \mathrm{s}^{-1}\right)\end{array}$ & $\begin{array}{c}{[\mathrm{Fe} / \mathrm{H}]} \\
(\mathrm{dex})\end{array}$ & $\begin{array}{c}{[\mathrm{Fe} \mathrm{I} / \mathrm{H}]} \\
(\mathrm{dex})\end{array}$ & $\begin{array}{c}{[\mathrm{Si} / \mathrm{H}]} \\
(\mathrm{dex})\end{array}$ & $\begin{array}{c}{[\mathrm{Ca} / \mathrm{H}]} \\
(\mathrm{dex})\end{array}$ & $\begin{array}{c}{[\mathrm{Ni} / \mathrm{H}]} \\
(\mathrm{dex})\end{array}$ & $\begin{array}{c}{[\mathrm{Eu} / \mathrm{H}]} \\
(\mathrm{dex})\end{array}$ \\
\hline 5900 & 1.4 & 4.1 & $-0.26 \pm 0.06(34)$ & $-0.24 \pm 0.06(11)$ & $-0.22 \pm 0.06(6)$ & $-0.25 \pm 0.04(6)$ & $-0.42 \pm 0.08(7)$ & $+0.61 \pm 0.07(5)$ \\
\hline
\end{tabular}

Notes. The standard deviation and the number of lines used in the analysis are also given.

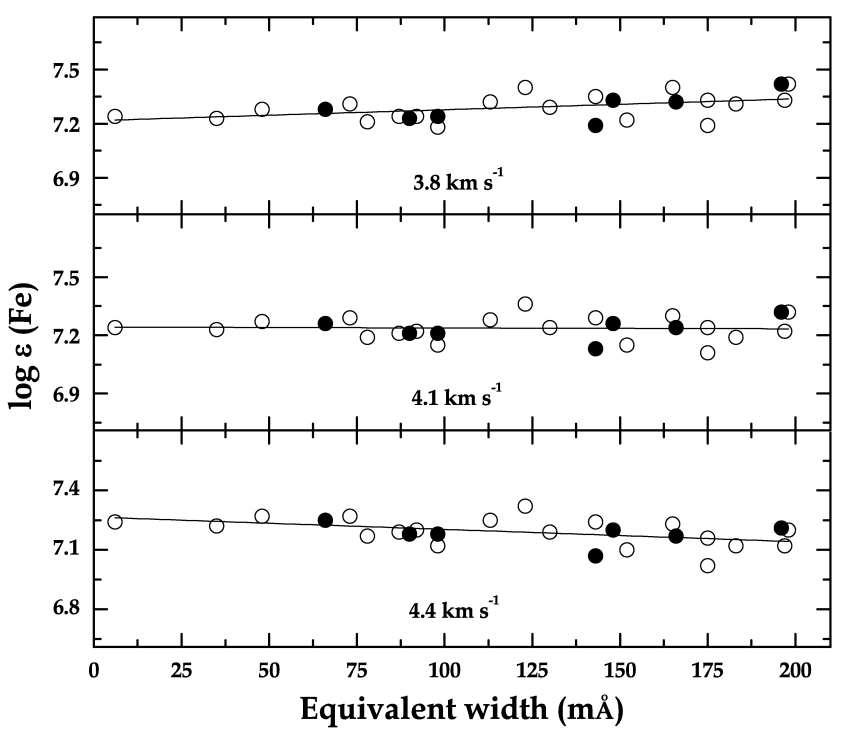

Fig. 8. Abundances derived from the Fe I and Fe II (neutral lines - open circles; ionized lines - filled circles) lines in red wavelength region plotted vs. the EW of the line, illustrating the estimation of the microturbulent velocity for HD 50975A.

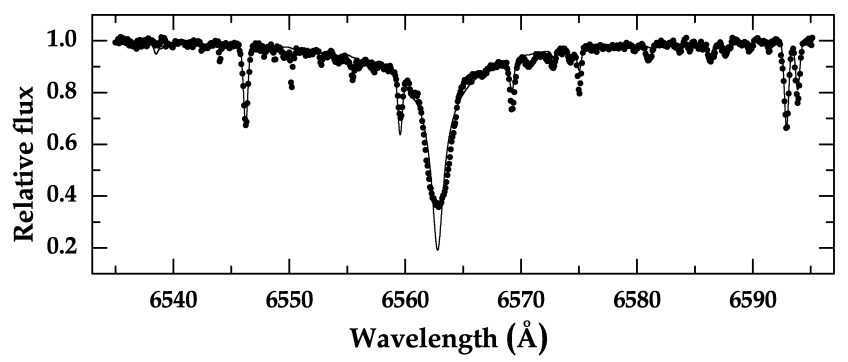

Fig. 9. Observed spectrum of HD 50975 (dots) around $\mathrm{H}_{\alpha}$ line, along with the one calculated using atmospheric model with $T_{\text {eff }}=6000 \mathrm{~K}$, $\log (g)=1.5(\mathrm{cgs})$, and $[\mathrm{Fe} / \mathrm{H}]=-0.3$.

laboratory measurements. The VALD database (Piskunov et al. 1995) was used to examine some problematic lines. In Table 4 the EWs are given for measured lines, along with the oscillator strengths and excitation potentials. The resulting mean relative abundances normalized with the solar-system abundances of Grevesse \& Sauval (1998) are listed in Table 3. The abundance $[X / H]$ is determined as the difference between the $X$ abundance of the star and the Sun, i.e. $[X / H]=\log \epsilon_{\star}(X)-\log \epsilon_{\odot}(X)$, where the abundance $\log \epsilon$ corresponds to the standard logarithmic scale with the hydrogen abundance $\log \epsilon(\mathrm{H})=12.00$. Some wavelength regions around important atomic lines were calculated using the spectral synthesis code STARSP (Tsymbal 1996) based on the Kurucz's code SYNTHE. The code for the spectrum synthesis adopts atomic and molecular partition functions and the continuous opacity package from ATLAS9 (Kurucz 1992).
The systematic errors in abundances produced by the uncertainties in $T_{\text {eff }}( \pm 150 \mathrm{~K}), \log g( \pm 0.3 \mathrm{dex})$, and microturbulent velocity $\left( \pm 0.3 \mathrm{~km} \mathrm{~s}^{-1}\right)$ would lead to errors, less than $0.15 \mathrm{dex}$ for the calculated elements.

The amount of veiling of EWs of the primary star because the continuum of the secondary star was examined on the basis of iron abundance calculated using Fe I lines over a broad wavelength region from 4000 to $9600 \AA$. A significant trend in the iron abundance from the wavelength of the line used was not detected in the region from 6100 to $9600 \AA$, $[\mathrm{Fe} / \mathrm{H}]=-0.26 \pm$ 0.06 dex, which agrees with the modeled fluxes of the companion stars. Indeed, it is difficult to detect minor $\sim 3 \%$ variations in the flux in the observed spectrum because of uncertainties in atomic data, setting of continuum, and measurements of EWs. However, the mean iron abundance calculated using lines in the wavelength region from 5400 to $5600 \AA$ (around $\lambda_{\text {eff }}$ of $V$ band) was found to be lower, $[\mathrm{Fe} / \mathrm{H}]=-0.36 \pm 0.04$ dex (9 lines), which confirms the increasing flux from the secondary star and reduction of EWs of HD 50975. The continuum definition is perfect in the visual spectral region, and the difference of 0.1 dex in the mean abundance is because of lower EWs by about $5 \%$ on average. Two other samples of iron lines in the wavelength regions from 5030 to $5230 \AA$ and from 4150 to $4260 \AA$ give the mean iron abundance of $[\mathrm{Fe} / \mathrm{H}]=-0.48 \pm 0.04$ dex (12 lines) and $[\mathrm{Fe} / \mathrm{H}] \simeq-1.0 \pm 0.14 \mathrm{dex}$ (3 lines), respectively, in support of increasing veiling of EWs for HD 50975A in the blue spectral region. Unfortunately, in the blue region shortward of $4300 \AA$, the continuum definition is uncertain and a number of unblended lines are low, so the calculated mean iron abundance is uncertain.

\section{Mass and luminosity}

The mass and bolometric magnitude of the secondary star HD 50975B was accepted to be a mean value for B2 mainsequence stars according Harmanec (1988), $M_{\mathrm{B}}=8.6 M_{\odot}$ and $M_{\text {bol }}=-4.5 \mathrm{mag}$. The adopted mass for the secondary is close to what was derived from calibration given by Schmidt-Kaller (1982). The maximum (upper) mass of the primary star HD 50975A was estimated using the mass of secondary star, the mass function, and orbital inclination $i \leq 80 \mathrm{deg}$, $M_{\mathrm{A}} \leq 12.7 M_{\odot}$. The inclination angle of $80 \mathrm{deg}$ was adopted because the light curve does not show any indication of eclipses, and it takes the theoretical dimensions of both stars and the orbit into account. On the other hand, the minimal mass of the primary HD 50975A should be higher than the mass of the secondary star because of its more advanced stage of evolution.

An independent determination of the bolometric magnitude for HD 50975A can be made with the well known relation $\log \left(M / M_{\odot}\right)=\log (g)-4 \log \left(T_{\text {eff }}\right)-0.4 M_{\text {bol }}+12.51$, if the gravity and temperature are known from spectroscopy. When using the effective temperature $T_{\text {eff }}=5900 \mathrm{~K}$ and adopting a spectroscopic gravity of $\log g=1.4$, the relation leads to the bolometric 
Table 4. Wavelengths, excitation potentials, gf-values, and equivalent widths of lines measured in the spectrum of HD 50975A.

\begin{tabular}{|c|c|c|c|c|c|c|c|}
\hline Wavelength $(\AA)$ & $\mathrm{LEP}(\mathrm{eV})$ & $\log g f$ & $E W(\mathrm{~m} \AA)$ & Wavelength $(\AA)$ & $\operatorname{LEP}(\mathrm{eV})$ & $\log g f$ & $E W(\mathrm{~m} \AA)$ \\
\hline Si I & & & & Fe I & & & \\
\hline 6155.14 & 5.62 & -0.97 & 83 & 7068.42 & 4.08 & -1.38 & 62 \\
\hline 6237.33 & 5.61 & -1.14 & 58 & 7090.40 & 4.23 & -1.21 & 63 \\
\hline 7165.58 & 5.87 & -0.54 & 88 & 7130.94 & 4.22 & -0.79 & 104 \\
\hline 7405.79 & 5.61 & -0.54 & 121 & 7583.80 & 3.02 & -1.99 & 94 \\
\hline 8742.47 & 5.87 & -0.51 & 122 & 8327.05 & 2.20 & -1.53 & 259 \\
\hline 8892.72 & 5.98 & -0.83 & 81 & 8468.40 & 2.22 & -2.07 & 213 \\
\hline $\mathrm{Ca} \mathrm{I}$ & & & & 8514.07 & 2.20 & -2.23 & 181 \\
\hline 6166.44 & 2.52 & -1.14 & 78 & 8611.81 & 2.84 & -1.90 & 154 \\
\hline 6439.08 & 2.52 & 0.39 & 232 & 8674.75 & 2.83 & -1.85 & 172 \\
\hline 6449.81 & 2.52 & -0.50 & 150 & 8757.19 & 2.84 & -2.03 & 125 \\
\hline 6471.67 & 2.52 & -0.69 & 121 & 8838.43 & 2.86 & -1.98 & 138 \\
\hline 6493.79 & 2.52 & -0.11 & 187 & Fe II & & & \\
\hline 6499.65 & 2.52 & -0.82 & 105 & 6084.10 & 3.20 & -3.98 & 98 \\
\hline $\mathrm{Fe} I$ & & & & 6113.33 & 3.22 & -4.31 & 66 \\
\hline 6055.99 & 4.73 & -0.46 & 78 & 6149.25 & 3.89 & -2.92 & 148 \\
\hline 6065.48 & 2.61 & -1.53 & 175 & 6247.55 & 3.89 & -2.51 & 196 \\
\hline 6151.62 & 2.18 & -3.30 & 48 & 6369.46 & 2.89 & -4.36 & 90 \\
\hline 6159.41 & 4.61 & -1.97 & 6 & 6416.92 & 3.89 & -2.85 & 143 \\
\hline 6165.37 & 4.14 & -1.55 & 35 & 6432.68 & 2.89 & -3.74 & 166 \\
\hline 6200.32 & 2.61 & -2.44 & 87 & 7222.39 & 3.89 & -3.49 & 85 \\
\hline 6213.43 & 2.22 & -2.66 & 113 & 7224.47 & 3.89 & -3.40 & 99 \\
\hline 6219.28 & 2.20 & -2.43 & 143 & 7479.69 & 3.89 & -3.88 & 56 \\
\hline 6322.69 & 2.59 & -2.43 & 92 & 7711.71 & 3.90 & -2.74 & 189 \\
\hline 6335.34 & 2.20 & -2.23 & 152 & $\mathrm{Ni} I$ & & & \\
\hline 6336.84 & 3.69 & -1.05 & 130 & 6767.77 & 1.83 & -2.17 & 106 \\
\hline 6355.04 & 2.84 & -2.42 & 73 & 6772.36 & 3.66 & -0.98 & 43 \\
\hline 6393.60 & 2.43 & -1.62 & 197 & 7422.30 & 3.63 & -0.14 & 115 \\
\hline 6411.65 & 3.65 & -0.82 & 165 & 7522.78 & 3.66 & -0.33 & 91 \\
\hline 6419.98 & 4.73 & -0.24 & 98 & 7525.12 & 3.63 & -0.56 & 87 \\
\hline 6421.35 & 2.28 & -2.03 & 175 & 7574.05 & 3.83 & -0.55 & 55 \\
\hline 6430.85 & 2.18 & -2.01 & 183 & 7727.66 & 3.68 & -0.17 & 114 \\
\hline 6663.45 & 2.42 & -2.48 & 123 & Eu II & & & \\
\hline 6677.99 & 2.69 & -1.47 & 198 & 6049.51 & 1.28 & -0.80 & 31 \\
\hline 6750.15 & 2.42 & -2.62 & 98 & 6437.64 & 1.32 & -0.28 & 72 \\
\hline 6820.37 & 4.64 & -1.32 & 30 & 7077.22 & 1.25 & -0.64 & 62 \\
\hline 6841.35 & 4.61 & -0.75 & 74 & 7370.22 & 1.32 & -0.29 & 98 \\
\hline 6843.67 & 4.55 & -0.93 & 56 & 7426.57 & 1.28 & -0.67 & 54 \\
\hline
\end{tabular}

magnitude, $M_{\mathrm{bol}}=-5.5 \pm 0.3 \mathrm{mag}$, for the primary star in the case of mass $8.6 M_{\odot} \leq M_{\mathrm{A}} \leq 12.7 M_{\odot}$.

Extremely strong oxygen lines of an infrared triplet at 7771.94, 7774.17, and 7775.39 $\AA$ in the spectrum of HD 50975A confirm high luminosity for the primary star. The EW of the line at $7771.94 \AA$, which is the least blended member of the triplet, was measured to be about $600 \mathrm{~m} \AA$, and the combined EW of all three components $E W=1.6 \AA$ agrees with luminosity around $\log L / L_{\odot} \simeq 4$ (Kovtyukh et al. 2008).

The final parameters for the both components of HD 50975 are provided in Table 5. The mean efective temperature of B2 main sequence stars according to De Jager \& Nieuwenhuijzen (1987) was accepted for HD 50975B. Uncertainties are evaluated in accordance with the uncertainty of \pm 0.5 in the spectral subtype for secondary component, $\pm 0.5 \mathrm{mag}$ in the difference between magnitudes $\Delta V$, and \pm 0.05 mag in $E(B-V)$. In Fig. 10 the positions of both binary components in the Hertzsprung-Russell (HR) diagram are compared to the evolutionary tracks calculated by Padova group (see Bertelli et al. 2009). Two tracks are given for $8 M_{\odot}$ and $10 M_{\odot}$ with a solar and a depleted metallicity of $Z=0.008([\mathrm{Fe} / \mathrm{H}]=-0.32 \mathrm{dex})$ to illustrate the uncertainty. In addition, a sample of F-G type stars adopted from Kovtyukh et al. (2010) is shown to display
Table 5. Fundamental parameters for the primary and secondary components of HD 50975A.

\begin{tabular}{cccccc}
\hline \hline Star & $\begin{array}{c}\text { Sp } \\
\text { type }\end{array}$ & $\begin{array}{c}T_{\text {eff }} \\
(\mathrm{K})\end{array}$ & $\begin{array}{c}\text { Mass } \\
\left(M_{\odot}\right)\end{array}$ & $\begin{array}{c}M_{\text {bol }} \\
(\mathrm{mag})\end{array}$ & $\log L / L_{\odot}$ \\
\hline $\mathrm{A}$ & $\mathrm{F} 8 \mathrm{Ib}$ & $5900 \pm 150$ & $10.7 \pm 2.0$ & $-5.5 \pm 0.3$ & $4.1 \pm 0.1$ \\
$\mathrm{~B}$ & $\mathrm{~B} 2$ & $20900 \pm 2500$ & $8.6 \pm 0.6$ & $-4.5 \pm 0.2$ & $3.7 \pm 0.1$ \\
\hline
\end{tabular}

the area occupied by single F supergiants of different masses in the HR diagram: open circles are their original $M_{V}$, crosses $-M_{V}$ derived from trigonometric parallaxes. As can be seen, the initial mass of the primary star HD 50975A is about $10 M_{\odot}$ according the determined stellar parameters and evolutionary tracks. The isochrone of $25 \mathrm{Myr}$ fits both components of HD 50975 well in the $\log \left(T_{\text {eff }}\right)$ vs. $\log \left(L / L_{\odot}\right)$ plane (see Fig. 10).

\section{Discussion and conclusions}

A comprehensive analysis of the long-term radial velocity measurements collected for the primary star of a spectroscopic binary HD 50975A was performed with the goal of calculating orbital elements. The orbital period was found to be $190.22 \pm$ 0.01 days, and the mass function was calculated to be high, 


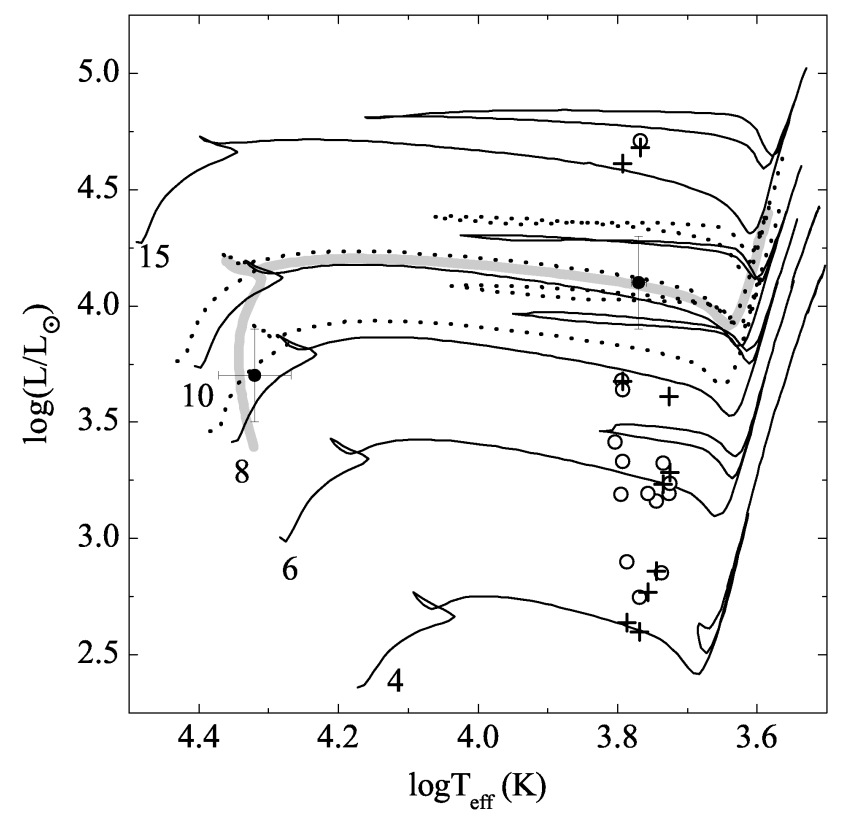

Fig. 10. Position of the primary and secondary components of HD 50975 (filled circles) in the $\log \left(T_{\text {eff }}\right)$ vs. $\log \left(L / L_{\odot}\right)$ plane. Evolutionary tracks retrieved from Bertelli et al. (2009) labeled by their initial mass in solar units are given for solar composition $(Z=0.019$, thin solid line) and depleted metallicity $(Z=0.008$, dotted line). The thick gray line presents a segment of the $25 \mathrm{Myr}$ isochrone. A sample of single F-G type stars are plotted for comparison.

$f(m)=1.402 \pm 0.005 M_{\odot}$. The absence of eclipses in the light curve gives evidence about an orbital inclination of $i \leq$ $80 \mathrm{deg}$. Unfortunately, high-resolution spectroscopy shortward of $3600 \AA$ and a search for absorption lines of the secondary star were unsuccessful. Therefore, calculating the mass ratio was postponed to the future.

Analysis and modeling of the low-resolution IUE spectrum of HD 50975 in the far ultraviolet region, along with those for early type standard stars, revealed the nature of the hot secondary. HD 50975B is a star of spectral type B2 \pm 0.5 near ZAMS with an effective temperature $T_{\text {eff }} \simeq 21000 \mathrm{~K}$. Comparison of the SED for HD 50975 with those for F8 supergiant and hot secondary confirm the spectral type about B1 for the secondary star HD 50975B. The mass and bolometric magnitude was adopted to be $M \simeq 8.6 M_{\odot}$ and $M_{\text {bol }} \simeq-4.5 \mathrm{mag}$, according to the mean parameters for B2 main sequence stars (Harmanec 1988). Using $M_{\mathrm{bol}}=-4.5 \mathrm{mag}$ and $\mathrm{BC}=-1.5 \mathrm{mag}$ (see Flower 1996), $M_{V} \simeq-3.0 \mathrm{mag}$ for HD 50975B.

The high-resolution spectrum of HD 50975 in the large spectral region was studied in detail with the goal of estimating the atmospheric and fundamental parameters of both stellar components. The red and near infrared spectral region from 6100 to $9600 \AA$ was analyzed and modeled using the method of atmospheric models with the goal of estimating atmospheric parameters, abundances, luminosity, and distance for the primary star HD 50975A. The effective temperature and surface gravity were found to be typical of F8 supergiants: $T_{\text {eff }}=5900 \pm 150 \mathrm{~K}$, and $\log (g)=1.4 \pm 0.3$ (cgs). The spectroscopic temperature and gravity lead to a bolometric magnitude for the primary star, $M_{\text {bol }}=-5.5 \pm 0.3 \mathrm{mag}$ for mass $8.6 M_{\odot} \leq M_{\mathrm{A}} \leq 12.7 M_{\odot}$. Strong lines of the oxygen infrared triplet confirm high luminosity. The spectroscopic parameters compared with stellar evolutionary tracks comfirm the initial mass of about $10 M_{\odot}$ for the primary. The distance modulus, $\left(V-M_{V}\right)=13.0 \mathrm{mag}$, and total absorption, $A_{V}=0.3 \mathrm{mag}$, places HD 50975 at the distance of $3.5 \mathrm{kpc}$. Using the estimated distance and galactic longitude, $b=+5 \mathrm{deg}$, the distance was estimated to be about $300 \mathrm{pc}$ above the Galactic plane. The iron abundance calculated using equivalent widths and atmospheric models confirms slight metal deficiency in the atmosphere of primary, $[\mathrm{Fe} / \mathrm{H}]=-0.26$ dex. Modeling of the flux of the both companion stars and of the measurements of veiled EWs of iron lines give evidence for a minor contribution of secondary HD 50975B in the red and near infrared spectral region. The calculated abundances for $\mathrm{Si}$ and $\mathrm{Ca}$ agree with the mild iron deficiency on average. The iron abundances for single A, F, and G-supergiants with the masses between 4 and $15 M_{\odot}$ are between $\log \epsilon(\mathrm{Fe})=7.28$ and 7.67 in comparison with 7.45 for the Sun (Lyubimkov et al. 2010). The abundance of europium was found to be enhanced in the atmosphere of HD 50975A, $[\mathrm{Eu} / \mathrm{H}]=+0.61 \pm 0.07$ dex. Thus, the site where HD 50975 formed seems to be enriched by r-process elements in the past.

The absolute magnitude $M_{V}=-3.0$ mag for HD 50975B and the distance modulus estimated for HD 50975A lead to the apparent magnitude $V \simeq 10.0 \mathrm{mag}$ for the secondary star HD 50975B in the $V$ band, therefore the difference in magnitudes between the secondary and primary stars could be about $2.5 \mathrm{mag}$. Detected veiling of EW by about $5 \%$ around $\lambda_{\mathrm{eff}}=$ $5450 \AA$ of $V$ band confirms a bit larger difference in the apparent magnitudes. Some discrepancy between the estimations on the basis of CCF and photometry seems to come from the different wavelength range for the CORAVEL mask and from the neglected slight metal deficiency for HD 50975A.

High luminosity, $\log \left(L / L_{\odot}\right)=4.1 \pm 0.1$, places HD 50975A among the brightest yellow supergiants. Unfortunately, the parallax of HD 50975, $\pi=2.68 \pm 0.54$ mas (van Leeuwen 2007), leads to the distance of $0.37_{-0.06}^{+0.09} \mathrm{kpc}$. At such a distance, $\log \left(L / L_{\odot}\right)=2.5$ for the primary star HD 50975A and 1.1 for the secondary star HD 50975B, which are discrepant with the results gathered from spectroscopy. In addition, the minimum mass of secondary, which matches the calculated value of $f(m)=$ $1.40 M_{\odot}$, is about $6 M_{\odot}$ in the case of $M_{\mathrm{A}}>M_{\mathrm{B}}$ and $i<80 \mathrm{deg}$. On the other hand, the position of HD 50975 at the distance of $0.37 \mathrm{kpc}$ should be variable because of the orbital motion and a change in the position during a year should exceed the parallax. Curiously, the old HIPPARCos parallax, $\pi=1.38 \pm 0.98$ mas (Perryman \& ESA 1997), does not contradict the high luminosity estimated from spectroscopy. We concluded that the parallax and proper motions are uncertain for HD 50975A.

The progenitor of the SN 2011dh was detected in archival pre-explosion Hubble Space Telescope images (see Maund et al. 2011). It is consistent with an F8 supergiant $\left(\log \left(L / L_{\odot}\right)=4.92 \pm\right.$ $\left.0.2, T_{\text {eff }}=6000 \pm 280 \mathrm{~K}\right)$. Through comparison with stellar evolution tracks, this corresponds to a single star at the end of core C-burning with an initial mass of $13 \pm 3 M_{\odot}$. Early photometric and spectroscopic observations show fast evolution similar to the transitional type IIb SN 2008 ax and suggest that a large amount of the progenitor's hydrogen envelope was removed before explosion. There are two main mechanisms proposed for stars to lose their outer layers: strong winds and mass transfer in interacting binary systems. Bersten et al. (2012) carried out stellar evolution calculations for a system of two massive stars in a close orbit, in which phases of mass transfer occur. By assuming a system of stars with initial masses of 16 and $10 M_{\odot}$, and an initial period of 125 days, they were able to obtain a configuration for the mass-donor star that closely matches the observations in the pre-supernova images and has a core mass that is consistent with their hydrodynamic model. The parameters of the binary 
system HD 50975 are not very far from those simulated for the SN 2011dh progenitor. HD 50975 is near a semi-detached configuration with a radius of primary of $R_{\mathrm{A}}=107 R_{\odot}$, estimated using the fundamental relation among $R, L$, and $T_{\text {eff }}$, and a distance between both components of $370 R_{\odot}$, calculated using the period and masses given in Tables 2 and 5. The radius of the Roche lobe for HD 50975A was calculated to be $120 R_{\odot}$ according to a formula by Eggleton (1983). Double-peaked light curve of HD 50975A, which is synhronized with the maximum and minimum of radial velocity variations, confirms a gravitational distortion of the primary star.

Acknowledgements. L.Z. acknowledges the support from the Research Council of the Lithuania under the grant No.VIZIT-5-TYR-052. We thank S. Velichko and A. Bondar for observations on the $2 \mathrm{~m}$ telescope at the observatory on the Terskol Peak. The referee, T. Zwitter, is thanked for his constructive comments on the paper. This research made use of the SIMBAD database, operated at the CDS, Strasbourg, France. Some of the data presented in this paper were obtained from the Multimission Archive at the Space Telescope Science Institute (MAST). STScI is operated by the Association of Universities for Research in Astronomy, Inc., under NASA contract NAS5-26555. Support for MAST for non-HST data is provided by the NASA Office of Space Science via grant NAG57584 and by other grants and contracts.

\section{References}

Barbier, R., Dossin, F., Jaschek, M., et al. 1978, A\&A, 66, L9

Bartkevicius, A., \& Sperauskas, J. 1994, Balt. Astron., 3, 49

Bersten, M. C., Benvenuto, O. G., Nomoto, K. E., et al. 2012, ApJ, 757, 31

Benvenuto, O. G., Bersten, M. C., \& Nomoto, K. 2013, ApJ, 762, 74

Bertelli, G., Nasi, E., Girardi, L., \& Marigo, P. 2009, A\&A, 508, 355

Crawford. D. L. 1975, AJ, 80, 955

Crawford. D. L. 1978, AJ, 83, 48

Crawford, D. L., \& Mandwewala, N. 1976, PASP, 86, 91

De Jager, C., \& Nieuwenhuijzen, H. 1987, A\&A, 177, 217

Eggleton, P. P. 1983, ApJ, 268, 368

Flower, J. P. 1996, ApJ, 469, 355

Gray, R. O. 1991, A\&A, 252, 237
Gray, R. O. 1998, AJ, 116, 482

Gray, R. O., Napier, M. G., \& Winkler, L. I. 2001, AJ, 121, 2148

Grevesse, N., \& Souval, A. J. 1998, Space Sci. Rev., 85, 161

Griffin, R. 1967, ApJ, 148, 465

Harmanec, P. 1988, Bull. Astron. Inst. Czechoslovakia, 39, 329

Hauck, B., \& Mermilliod, M. 1998, A\&AS, 129, 431

Koen, C., \& Eyer, L. 2002, MNRAS, 331, 45

Kovtyukh, V. V., Chekhonadskikh, F. A., Luck, R. E., et al. 2010, MNRAS, 408 1568

Kovtyukh, V. V., Gorlova, N. I., \& Belik, S. I. 2012, MNRAS, 423, 3268

Kurucz, R. L.1992, in Stellar Populations of Galaxies, eds. B. Barbuy, \& A. Renzini (Dordrecht: Kluwer), IAU Symp., 149, 225

Kurucz, R. L. 1993, CD-ROM 13 \& 18, Smithsonian Astrophys. Obs

Lyubimkov, L. S., Lambert, D. L., Rostopchin, S. I., Rachkovskaya, T. M., \& Poklad, D. B. 2010, MNRAS, 402, 1369

MacConnell, D. J., \& Bidelman, W. P. 1976, AJ, 81, 225

Massa, D., \& Fitzpatrick, E. L. 2000, ApJS, 126, 517

Maund, J. R., Fraser, M., Ergon, M., et al. 2011, ApJ, 739, L37

Munari, U., \& Zwitter, T. 1997, A\&A, 318, 269

Nidever, D. L., Marcy, G. W., Butler, R. P., Fische, D. A., \& Vogt, S. S. 2002, ApJS, 141, 503

Olsen, E. H. 1979, A\&AS, 37, 367

Olsen, E. H. 1983, A\&AS, 54, 55

Perryman, M. A. C., \& ESA 1997, The HIPPARcos and Tycho catalogues (Noordwijk, Netherlands: ESA Publications Division), ESA SP, 1200

Piskunov, N. E., Kupka, F., Ryabchikova, T. A., Weiss, W. W., \& Jeffery, C. S. 1995, A\&AS, 112, 525

Pojmanski, G. 2002, Acta Astron., 52, 397

Rountree, J., \& Sonneborn, G. 1991, ApJ, 369, 515

Schmidt-Kaler, T. 1982, in Landolt-Boernstein, Vol. VI/2b, eds. K. Schaifers, \& H. H. Voigt (Springer-Verlag), 1

Shulov, O. S. 1986, Sov. Astron., 30, 435

Sviderskiene, Z. 1988, Vilnius Obs. Bull., 80, 7

Tsymbal,V. V. 1996, ASP Conf. Ser., 108, 198

Udry, S., Mayor, M., Maurice, E., et al. 1999, in Precise Stellar Radial Velocities, eds. J. B. Hearnshaw, \& C. D. Scarfe, ASP Conf., 185, 383

Upgren, A., Sperauskas, J., \& Boyle, R. P. 2002, Balt. Astron., 11, 91

van Leeuwen, F. 2007, A\&A, 474, 653

Vieira, E. F., \& Ponz, J. D. 1995, A\&AS, 111, 393 